\title{
Hand Movement Based Message Transmission and Body Physical Parameters Remote Monitoring Device for Disabled People
}

\author{
D Kalaiyarasi ${ }^{\mathrm{a}, 1}$, Shruthi.V $\mathrm{V}^{\mathrm{b}}$, VishnuPraba.A $^{\mathrm{b}}$, Vanaja.S $^{\mathrm{b}}$ \\ ${ }^{a}$ Associate Professor, Dept of ECE, Panimalar Engineering College, Chennai, India \\ ${ }^{b} U G$ scholar, Dept of ECE, Panimalar Engineering College, Chennai, India
}

\begin{abstract}
The main focus of the proposed model is to manifest a system that is reliable and also effective interms of communication between Disabled/paralyzed people with the caretaker. When it comes to looking after the disabled or paralytic people who are in large numbers such as in old age homes, Non-Governmental Organizations, and hospitals, it is considerably difficult to monitor the patients based on their needs immediately. To overcome this difficulty a simple yet effective module is developed to establish the communication between the patient (Transmitter) and caretaker (receiver) by mounting a device on the hand of the patient so that by simple movements made using the hand aspecific message is communicated to the device which is in a form of a board consisting of the LCD screen and voice module to indicate the message and the board is held by the caretaker separately. The transmission mode is wireless and the range is about 10 meters. The physical parameters of the patient's body are measured and monitored remotely. When there is any abnormal pattern in the parameters such as pulse rate and temperature it is indicated to the caretaker immediately.
\end{abstract}

Keywords. Arduino, Accelerometer, ZigBee, Pulse Rate sensor, temperature sensor.

\section{Introduction}

Embedded systems have extended their application in almost every aspect according to human needs. One such important application of Embedded systems is in healthcare. There are many kinds of research going to date in healthcare monitoring to provide effective outcomes in terms of health monitoring.

${ }^{1}$ Kalaiyarasi. D, Associateprofessor,Dept. of ECE, Panimalar Engineering College, Chennai; E-mail:id:kalaiccarthi@gmail.com 
Using the advancements made in the embedded systems the doctors can monitor the patient's health remotely using telecommunication and evaluate the effectiveness using the embedded trials. The module which we have designed and developed helps the spastic people to make their message conveyed effortlessly without much difficulty. The job of caretaker also becomes easy. The hand movement-based message transmission and body physical parameters remote indication device for spastic people helps both the patient as well as the caretaker equally. When the patient is in danger the pulse rate and the temperature is abnormal. This abnormality is detected and the message is conveyed to the caretaker immediately. Accelerometer plays a major role in determining the tilt direction based on $\mathrm{X}, \mathrm{Y}$, and $\mathrm{Z}$ directions and the angle of tilt made using the hand. And based on the tilt direction the specific message is conveyed.

The transmitting device consists of a ZigBee module, it is used because of its lowcost implementation, low power consumption and it is more effective because of its long battery life. The message is shown on the LCD screen and the message is also conveyed through the voice module which consists of a DFP layer mini mp3 player. With the help of the voice message the person who cannot read the message can easily be able to hear the message conveyed by the patient. The main goal of the proposed module is to replace the conventional method of communication between the patient and caretaker with modern technologies that are much faster and reliable.

\section{Literature review}

Mahaveer Penna, et. al [1] conferred the message transmission through motion recognition with the help of an accelerometer measuring the static acceleration of gravity in the tilt sensing requirements and dynamic acceleration causes due to motion, Vibration, and shock. Rafiqul Zaman Khan and Noor Adnan Ibraheem [2] discussed the hand gesture recognition process and how effectively it interacts with the machine. And also discussed the difficulties of hand gesture recognition systems. Ekta Pandey, et.al [3] discussed the motion-based message conveyor for disabled people using an Arduino UNO controller with the temperature sensor and the algorithm for the sensing and transmission of message Via ZigBee. Xiaolei Zhong, Ru Yao, Chun Chen, and Yuanjing Zhu [4] discussed how the transmission capacity of ZigBee can be improved and has provided the solution for the improvement of the transmission range. One of the methods used to enhance the transmission capacity is by using the sensor nodes in a wireless medium. Weimeng Niu, et.al[5] conferred that MEMS are termed as microelectromechanical systems, utilizing the innovation known as small-scale fabrication technology. It has gaps, pit, channels, cantilevers, films and further more mirrors mechanical parts. The MEMS depends on silicon. Yusuf Abdullahi Badanasi [6] discussed the hardware components and the software used to program the Arduino board with a clear explanation on how to write and construct the required module. Rohini Bhilare, et.al [7]-[10] discussed the Motion-Based Message Conveyor for Patients Using the Arduino System and ZigBee. Monitoring the physical parameters of the patient when the parameter values are above or below the threshold levels.

The proposed model is developed for wireless communication between the patient and the nurse using ZigBee and has embedded various sensors such as an accelerometer which is used to measure the tilt direction, pulse rate sensor,temperature sensor to monitor the parameters. When it becomes low or high compared to the threshold level the voice-based and text-based indication is transmitted to the caretaker 
immediately. The range of ZigBee transmission is also increased using the wireless sensor nodes.

\section{Methodology}

The proposed model consists of two modules, the transmitter (TX) module, and the receiver (RX) module. The transmitter module is placed on the patient's movable part for example on the hand. The receiver module is kept with the caretaker. The transmitter module consists of the accelerometer ADXL335, Temperature sensor, and Pulse Rate sensor MAX30100, Arduino microcontroller unit, ZigBee/RF module. The Receiver module consists of an RF module, a Microcontroller, a16x2 LCD Display, a Voice module, Speaker. The accelerometer in the transmitter module works by measuring the static acceleration due to gravity and based on the static acceleration the angle at which the device is rotated concerning the earth is determined. Whenever a patient needs any help he conveys his need by just tilting the accelerometer in different directions. This acts as an input to the accelerometer while the output of it is in volts that are connected to the controller board which acts as the processing unit. In addition to this, a temperature sensor and a pulse rate sensor are placed at the transmitter module to monitor the temperature and the pulse rate in a particular interval of time. The block diagram representation of the transmitter module is shown in Figure1.The second module of the proposed model is the receiver module which is connected with the transmitted module through the Wireless ZigBee/RF module. The Receiver device consists of the RF module, microcontroller, 16x2 LCDscreen, and the voice module along with the speaker. Once the tilt direction is measured by the accelerometer, the specific message is transmitted to the receiver after the processing performed by the Arduino. The message is displayed on the LCD screen and a voice message is also produced at the receiver side using the voice module. The physical parameters of the body such as temperature and pulse rate are monitored at regular interval of time when there is any abnormality i.e. when the value of the measure is below the threshold value the immediate message with the value of the parameters is displayed on the LCD screen and the voice-based message is also sent. The block diagram of the receiver module is represented in Figure 2.

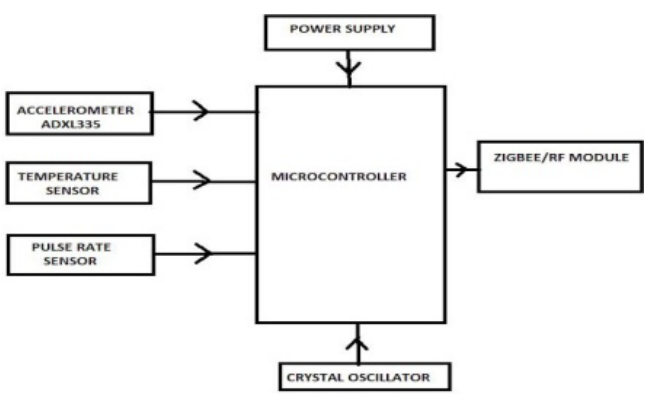

Figure 1. Block diagram of transmitter module

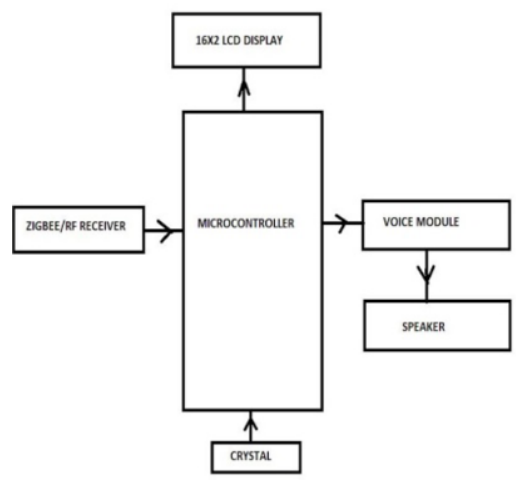

Figure 2.BlockDiagramoftheReceiverModule 


\section{Result}

As discussed in the methodology of the proposed model when the tilt direction is sensed using the accelerometer Concerning the earth and the data from the accelerometer is sent to the Arduino microcontroller for further processing and based on the tilt direction the specific message is transmitted to the receiver module using ZigBee and the physical parameters such as temperature and pulse rate is monitored remotely in regular intervals of time. When the value of the parameter goes below the threshold level then the message is sent to the caretaker that the patient is in an emergency. If the patient needs to communicate his needs immediately with the caretaker he just tilts his hand in that particular direction so that the message is conveyed both as text and voice to the caretaker. The proposed model setup of transmitter and receiver modules with the output delivered during the hand tilt to convey the message is shown in Figure 3 and the temperature and pulse rate monitoring module is shown in Figure4.
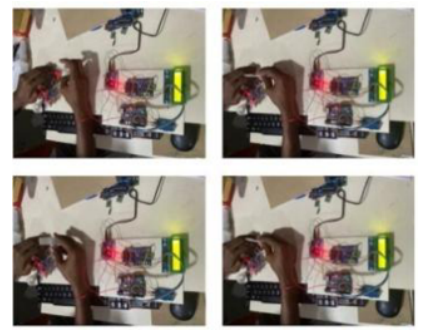

Figure 3. Different Messages conveyed for different tilt angles

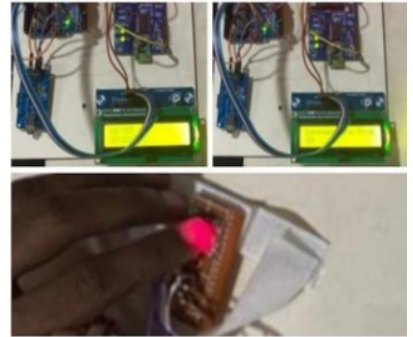

Figure 4. Pulse Rate and temperature monitoring module

\section{Future scope}

The range of transmission could be increased further and the number of physical parameters that are monitored is increased such as in addition to the temperature and pulse rate monitoring, the sugar level monitoring and oxygen level monitoring could be included and using the GSM module if the patient needs medical emergency the message along with the location of the patient is sent to the ambulance service center so that the ambulance can track the location easily and life of the patient can be saved.

\section{Conclusion}

The proposed module provides complete relief for the patients who are suffering from a disability of performing full-body movements in terms of easy communication with the caretaker to convey their needs immediately without much difficulty and if the patient's pulse rate and the temperature drops below the threshold level then the immediate message is sent to the caretaker so that the required measures can be taken. As the message is sent in both text and voice the caretaker who cannot read can easily understand. 


\section{Reference}

[1] MahaveerPennaet.al, hand gesture controlled message conveyer and controlling devices for physically disabled, $20183^{\text {rd }}$ IEEE International Conference on Recent Trends in Electronics, Information \& Communication Technology(RTEICT-2018), MAY18th \& $19^{\text {th }} 2018$.

[2] Rafiqul Zaman Khan and Noor AdnanIbraheem, hand gesture recognition: a literature review, International Journal of Artificial Intelligence \&Applications(IJAIA),Vol.3,No.4,July2012.

[3] EktaPandeyet.al.motion-based message conveyor for disabled patients.International Journal of Engineering Applied Sciences and Technology. 2020Vol.4, Issue12,ISSNNo.2455-2143,Pages240244.

[4] Xiaolei Zhong, RuYao, Chun Chenand YuanjingZhu.Research on Scalable ZigBee Wireless Sensor Network Expansion Solution. IOP Conf.Series: Materials Science and Engineering 394 (2018) 032071 doi:10.1088/1757-899X/394/3/032071.

[5] WeimengNiuet.al. Su mmary of Research Status and Application of MEMS Accelerometers. Journal of Computer and Communications, 2018, 6,215-221, ISSNOnline: 2327-5227.

[6] Yusuf Abdullahi Badanasi, https://doi.org/10.1109/ICECCO.2014.6997578, $11^{\text {th }}$ international conference on electronics, computer, and computation(ICECCO).

[7] RohiniBhilare, et.al, Motion Based Message Conveyor for Patient Using Arduino System and ZigBee, Volume5 Issue IV, April2017ICValue:45.98ISSN:2321-9653 International Journal for Research in Applied Science \& engineering technology.

[8] Leo Louis, Working principle of Arduino and using it as a tool for study and research, International Journal of Control, Automation, Communication and Systems(IJCACS),Vol.1,No.2,April2016.

[9] SumitTapkiret.al, Motion Based Message Conveyor for Physically Disabled People, International Research Journal of Engineering and Technology (IRJET)e-ISSN:2395-0056Volume:05Issue:03|Mar2018.

[10] Krushnapatiet.al, Motion Based Message Conveyor for Paralytic/Disabled Patient, ISSN:24552631 CApril2018IJSDR/Volume3,Issue4 